Annales Academiæ Scientiarum Fennicæ

Series A. I. Mathematica

Volumen 13, 1988, 183-189

\title{
A MARTINGALE APPROACH TO RELIABILITY THEORY: ON THE ROLE OF FILTRATION IN THE MODEL SPECIFICATION
}

\author{
E. Arjas and I. Norros
}

\section{Introduction}

Consider a device ("system") consisting of $k$ parts ("components"). The life lengths of the components form a non-negative random vector $\mathbf{S}=\left(S_{1}, \ldots, S_{k}\right)$. We assume that $\mathbf{S}$ is defined on a probability space $(\Omega, \mathcal{F}, P)$. The marked point process (MPP) $\left(T_{n}, I_{n}\right)_{n \geq 1}$ associated with $\mathbf{S}$ is defined as follows:

$$
\begin{gathered}
T_{1}=\inf _{1 \leq i \leq k} S_{i} ; \\
T_{n+1}=\inf \left\{S_{i}: 1 \leq i \leq k, S_{i}>T_{n}\right\}, \quad n \geq 1 ; \\
I_{n}=\left\{i: S_{i}=T_{n}\right\} .
\end{gathered}
$$

$\left(T_{n}\right)$ is then the ordered sequence of failure times and $\left(I_{n}\right)$ is the corresponding sequence of failure patterns.

Suppose now that the device is under observation ("monitored") in some way. A convenient mathematical model for this is a filtration (history) $\mathbf{F}=$ $\left(\mathcal{F}_{t}\right)_{t \geq 0}, \mathcal{F}_{t} \subseteq \mathcal{F}$, satisfying the "usual conditions" concerning right continuity and completeness.

Examples. Let $\mathbf{F}$ be the filtration generated by the marked point process $\left(T_{n}, I_{n}\right)$. This corresponds to the case where all component failures are immediately observed but no other stochastic information is available. This case is called component level monitoring. Another extreme case is system level monitoring, where the system failure time alone is observed. In this case, $\mathbf{F}$ is a filtration generated by a single stopping time.

Next we define a natural notion of hazard, based on a given type of monitoring. For each nonempty set $I \subseteq\{1, \ldots, k\}$ consider the counting process

$$
N_{t}(I)=\sum_{n \geq 1} 1_{\left\{T_{n} \leq t, I_{n}=I\right\}}
$$


This means that $N_{t}(I)=1$ if a failure with exactly the pattern $I$ occurs before or at $t$, and 0 otherwise. Suppose that $N_{t}(I)$ is F-adapted. Denote the Fcompensator of $N(I)$ by $A(I)$. The process $A(I)$ satisfies the following intuitive requirement for hazard:

$$
d A_{t}(I)=P\left(T_{n} \in d t, I_{n}=I \mid \mathcal{F}_{t-}\right) \text { for } t \in\left(T_{n-1}, T_{n}\right]
$$

Remarks. 1. If a process $N(I)$ is not $\mathbf{F}$-adapted, one can instead consider the $\mathbf{F}$-submartingale $E\left[N_{t}(I) \mid \mathcal{F}_{t}\right]$ and its compensator.

2. This formalism can easily be extended to cover other instantaneous events than failures, e.g., replacements, repairs etc.

\section{On the role of filtration in reliability modeling}

\subsection{Ageing}

The following strong form of ageing was defined in Arjas [1]. Denote by

$$
R_{t}=(\mathbf{S}-t \cdot \mathbf{1})^{+}
$$

the vector of residual component life lengths at time $t$ (the positive part is taken componentwise).

Definition. The random life length vector $\mathbf{S}$ is said to have increasing failure rate with respect to the filtration $\mathbf{F}$ (in short: $\mathbf{S}$ is $\mathbf{F}$-IFR) if the conditional distribution

$$
P\left(R_{t} \in \cdot \mid \mathcal{F}_{t}\right)
$$

is non-increasing in $t$ in the sense of stochastic ordering.

The intuitive meaning of increasing failure rate distributions is that older components have stochastically shorter residual lives. However, this interpretation is not quite straightforward. The example below shows that the choice of filtration is crucial.

Suppose that $\mathbf{S}$ is $\mathbf{F}$-IFR and that $\mathbf{G}=\left(\mathcal{G}_{t}\right)$ is another filtration such that $\mathcal{G}_{t} \subseteq \mathcal{F}_{t}$ for all $t$. Does this imply that $\mathbf{S}$ is $\mathbf{G}$-IFR? In particular, does IFR relative to component level monitoring imply IFR relative to system level monitoring?

The answer is "no", and there is a very simple counterexample. Indeed, the system consisting of two exponential components in parallel, which has been used to show that the class of IFR distributions is not closed under formation of systems of independent components (see Barlow and Proschan [4]), works also in our slightly different setting.

Example. Let $S_{1}$ and $S_{2}$ be independent and exponentially distributed with unequal parameters. Consider the parallel system consisting of these components, with system life length $\tau=S_{1} \vee S_{2}$. Now, if

$$
\mathcal{F}_{t}=\sigma\left\{1_{\left\{S_{1} \leq s\right\}}, 1_{\left\{S_{2} \leq s\right\}} ; s \leq t\right\}
$$


it is obvious that $\mathbf{S}$ is $\mathbf{F}$-IFR, and so is $\tau$. On the other hand, if

$$
\mathcal{G}_{t}=\sigma\left\{1_{\{\tau \leq s\}} ; s \leq t\right\},
$$

then $\tau$ is not G-IFR (which here reduces to the standard univariate notion of IFR).

Remark. The answer can be "yes" for weaker ageing properties, such as "new better than used (NBU) with respect to F", see Arjas [1].

\subsection{Dependence}

In this section, we consider "the dependence on the choice of history" of the following notion of positive dependence.

Definition (Arjas and Norros [2], Norros [7]). A system with component life length vector $\mathbf{S}$ is said to be weakened by failures (WBF) if the conditional distribution

$$
P\left(R_{t} \in \cdot \mid \mathcal{F}_{t}\right)
$$

jumps downwards at the failure times $S_{i}$, where $\mathbf{F}$ is the filtration generated by all component failure times $S_{i}$.

The intuitive meaning of this definition is that each failure causes a stochastic decrease of the lifetimes of the remaining components.

Remarks. 1. If $\mathbf{S}$ is IFR with respect to its internal (componentwise) history, then it is also weakened by failures. Indeed, the definition of IFR with respect to internal history includes the decrease of the conditional distribution of the residual lifetimes at failure times.

2. It can be shown that WBF implies the association of the random variables $S_{i}$. (See Arjas and Norros [2], Norros [7].)

Suppose that monotone subsystems are formed by components $S_{1}, \ldots, S_{k}$. For example, let

$$
\tau_{i}=\phi_{i}\left(S_{1}, \ldots, S_{k}\right), \quad i=1, \ldots, m,
$$

where the functions $\phi_{i}$ consist of successive $\vee$ - and $\wedge$-operations. Let $\mathbf{F}$ be the internal history of $\left(S_{1}, \ldots, S_{k}\right)$ and $\mathbf{G}$ that of $\left(\tau_{1}, \ldots, \tau_{m}\right)$. Clearly, $\mathbf{G}$ is a subhistory of $\mathbf{F}$. A special case is a partially observed system $\mathbf{S}$, for example, $m<k$ and $\tau_{i}=S_{i}, i=1, \ldots, m$.

Now we can ask the following question: if the system $\left(S_{1}, \ldots, S_{k}\right)$ is WBF, does it follow that the system $\left(\tau_{1}, \ldots, \tau_{m}\right)$ is also WBF (with respect to $\mathbf{G}$ )? As in the previous section, the answer is, somewhat surprisingly, negative. The following counterexample is based on the idea that the "positive message" that an unobservable component still works can be "encoded" in the failure time of an observable component. 
Example. Consider a system with three components $\mathbf{S}=\left(S_{1}, S_{2}, S_{3}\right)$ and let $\mathbf{F}=\left(\mathcal{F}_{t}\right)_{t \geq 0}$ be the internal history:

$$
\mathcal{F}_{t}=\sigma\left\{1_{\left\{S_{i} \leq s\right\}} ; i=1,2,3, s \leq t\right\} .
$$

Let the predictable $\mathbf{F}$-intensities (that is, derivatives of compensators) of $S_{1}, S_{2}$ and $S_{3}$ be given by

$$
\begin{aligned}
& \lambda_{t}(1)=1_{\left\{S_{1} \leq t\right\}} \\
& \lambda_{t}(2)=\left[1_{\left\{t \leq S_{1}\right\}}+2 \cdot 1_{\left\{t>S_{1}\right\}}\right] \cdot 1_{\left\{t \leq S_{2}\right\}} \\
& \lambda_{t}(3)= \\
& =\left[1_{\left\{t \wedge 1 \leq S_{1}\right\}} \cdot 1_{(1, \infty)}(t)+1_{\left\{t \wedge 1>S_{1}\right\}}\left(\frac{1}{1-S_{1}} \cdot 1_{\left(S_{1}, 1\right]}(t)+1_{(2, \infty)}(t)\right)\right] \cdot 1_{\left\{t \leq S_{3}\right\}} .
\end{aligned}
$$

It is straightforward to check that $\mathbf{S}$ is supportive (see Norros [8]), and this condition implies the WBF property. (Thus, this counterexample works also for supportivity.) The point is that if $S_{1}$ occurs during $(0,1)$, then the fact that $\lambda(3)$ vanishes on $(1,2]$ is compensated by its greater value $\left(1-S_{1}\right)^{-1}$ on $\left(S_{1}, 1\right]$.

Now consider the slightly coarser history $\mathbf{G}=\left(\mathcal{G}_{t}\right)_{t \geq 0}$, where only the failures of components 2 and 3 are monitored:

$$
\mathcal{G}_{t}=\sigma\left(1_{\left\{S_{i} \leq s\right\}} ; i=2,3, s \leq t\right)
$$

The G-intensity of $S_{2}$ is

$$
\hat{\lambda}_{s}(2)=\left[P\left(S_{1}>s \mid \mathcal{G}_{s}\right)+2 \cdot P\left(S_{1} \leq s \mid \mathcal{G}_{s}\right)\right] \cdot 1_{\left\{s \leq S_{2}\right\}} .
$$

To show that $\left(S_{2}, S_{3}\right)$ is not WBF it suffices to show that $P\left(S_{1} \leq s \mid \mathcal{G}_{s}\right)$ can jump strictly downwards at a time $t$ when $S_{3}$ occurs and $S_{2}$ has not yet occurred. Indeed, then the intensity $\hat{\lambda}(2)$ of $S_{2}$ jumps downwards, and the conditional distribution of $S_{2}$ does not decrease, as it would if $\left(S_{2}, S_{3}\right)$ were WBF. We show that this is the case on the set $\left\{S_{2}>S_{3} \in(1,2)\right\}$.

Let us denote by $p(s)$ the continuous deterministic function

$$
p(s)=P\left(S_{1}>s \mid S_{2}>s, S_{3}>s\right)=P\left(S_{1} \geq s \mid S_{2} \geq s, S_{3} \geq s\right),
$$

and let $\hat{\lambda}_{s}(3)$ be the G-intensity of $S_{3}$. Suppose that we are on the set $\left\{S_{2}>\right.$ $\left.S_{3} \in(1,2)\right\}$, and denote $S_{3}(\omega)=t \in(1,2)$. Then the jump of $P\left(S_{1}>s \mid \mathcal{G}_{s}\right)$ at $t$ is

$$
\begin{gathered}
P\left(S_{1}>t \mid S_{2}>t, S_{3}=t\right)-p(t-) \\
=\frac{P\left(S_{1} \geq t, S_{2} \geq t, S_{3} \geq t\right) \cdot P\left(S_{3} \in[t, t+d t] \mid S_{1} \geq t, S_{2} \geq t, S_{3} \geq t\right)}{P\left(S_{2} \geq t, S_{3} \geq t\right) \cdot P\left(S_{3} \in[t, t+d t] \mid S_{2} \geq t, S_{3} \geq t\right)}-p(t) \\
=p(t) \cdot \frac{1 \cdot d t}{\hat{\lambda}_{t}(3) d t}-p(t)=p(t)\left(\frac{1}{\hat{\lambda}_{t}(3)}-1\right)>0,
\end{gathered}
$$

since $\hat{\lambda}_{t}(3)<1$ (as a weighted average of 0 and 1). The infinitesimal Bayes formula used above can be established rigorously by filtering theory of marked point processes (see Brémaud [5], Chapter 4). 


\subsection{Transformation of hazard}

In this third section we study how the effects of certain transformations of the compensators depend on the choice of filtration. Let $S$ be a finite totally inaccessible (unpredictable) stopping time on a filtration $\mathbf{F}=\left(\mathcal{F}_{t}\right)_{t \geq 0}$ satisfying the usual conditions. Denote $N_{t}=1_{\{t \geq S\}}$ and let $A^{\mathbf{F}}$ be the F-compensator of $N$.

Example 1. "Proportional improvement". Let $\alpha$ be a number in the interval $(0,1)$. Define the probability measure $P_{\alpha}$ by

$$
\left(\frac{d P_{\alpha}}{d P}\right)_{\mathcal{F}_{t}}=\alpha^{N_{t}} e^{(1-\alpha) A_{t}^{\mathbf{F}}}
$$

It is well known that the $\mathbf{F}$-compensator of $N$ with respect to $P_{\alpha}$ is $\alpha A^{\mathbf{F}}$ (see Brémaud [5], Chapter 6). This can also be interpreted in the following way: if the hazard of $S$ is reduced by a factor $\alpha$ and nothing else is changed, then the resulting probability distribution is $P_{\alpha}$.

Example 2. "Minimal repair" Suppose that when $S$ appears, it is immediately "erased" and the history continues "as if nothing had happened". The second appearance of $S$ (interprete: second failure) is "accepted" normally. We have shown elsewhere (Norros [9], Theorem 2.1 with $n=1$ ) that this kind of "improvement" of $S$ corresponds to transforming the original measure $P$ into a probability $Q$ such that

$$
\left(\frac{d Q}{d P}\right)_{\mathcal{F}_{t}}=1-N_{t}+A_{t}^{\mathbf{F}}
$$

By Girsanov-type theorems for point processes (see Brémaud [5], or, for example, Arjas and Norros [3], Proposition 2), the $Q$-compensator of $S$ is

$$
B_{t}^{\mathbf{F}}=\int_{0}^{t} \frac{A_{s}^{\mathbf{F}}}{1+A_{s}^{\mathbf{F}}} d A_{s}^{\mathbf{F}}=A_{t}^{\mathbf{F}}-\ln \left(1+A_{t}^{\mathbf{F}}\right) .
$$

Extending from this, we consider transformations of the compensator $A^{\mathbf{F}}$ which are of the form

$$
B_{t}^{\mathbf{F}}=g\left(A_{t}^{\mathbf{F}}\right)=\int_{0}^{t} g^{\prime}\left(A_{s}^{\mathbf{F}}\right) d A_{s}^{\mathbf{F}},
$$

where $g: \mathbf{R}_{+} \longrightarrow \mathbf{R}_{+}$is differentiable and increasing and $g(0)=0, g(\infty)=\infty$.

Now we ask the question: how does the effect of this transformation on the distribution of $S$ depend on the choice of the filtration $\mathbf{F}$ ? Denote by $\mathbf{G}=\left(\mathcal{G}_{t}\right)_{t \geq 0}$ the minimal filtration

$$
\mathcal{G}_{t}=\sigma\left\{1_{\{S \leq s\}} ; s \leq t\right\}
$$


Let the function $g$ satisfy the above conditions and denote

$$
B_{t}^{\mathbf{F}}=g\left(A_{t}^{\mathbf{F}}\right), \quad B_{t}^{\mathbf{G}}=g\left(A_{t}^{\mathbf{G}}\right)
$$

Let $Q^{\mathbf{F}}$ and $Q^{\mathbf{G}}$ be the measures defined by

$$
\begin{aligned}
& \left(\frac{d Q^{\mathbf{F}}}{d P}\right)_{\mathcal{F}_{t}}=g^{\prime}\left(A_{t}^{\mathbf{F}}\right)^{N_{t}} e^{A_{t}^{\mathbf{F}}-B_{t}^{\mathbf{F}}} \\
& \left(\frac{d Q^{\mathbf{G}}}{d P}\right)_{\mathcal{G}_{t}}=g^{\prime}\left(A_{t}^{\mathbf{G}}\right)^{N_{t}} e^{A_{t}^{\mathbf{G}}-B_{t}^{\mathbf{G}}} .
\end{aligned}
$$

Our main result is the following.

Theorem (Arjas and Norros [3]). Suppose $g$ is such that the function

$$
x \mapsto e^{-g(-\ln x)}
$$

is concave for $x \in(0,1)$. Then $S$ is stochastically smaller under $Q^{\mathbf{F}}$ than under $Q^{\mathbf{G}}$.

Proof. Use Jensen's inequality and the fact that

$$
1_{\{S>t\}} e^{A_{t}^{\mathbf{F}}} d P
$$

is a probability measure on $\mathcal{F}_{t}$.

It is easy to check that both the proportional improvement and the minimal repair transformation satisfy the conditions of the theorem. The latter case has some practical significance. Indeed, it says that the usual way of modeling minimal repair (conditioning only on the minimal history) gives always a too optimistic result in comparison with the more complex model with a richer filtration. A more detailed discussion can be found in Arjas and Norros [3].

\section{Concluding remarks}

In traditional reliability theory there is much emphasis in classes of distributions, which typically describe ageing and dependence, and the preservation of the defining class property in transformations which correspond to "reliability operations". In the present approach the filtration is an integral part of the given definitions, and actual distribution theory is less central. Consequently, the question "What properties are preserved under reliability operations?" must be answered differently here.

As we have seen above, the operation of changing a bigger filtration into a smaller one, which means that some of the conditioning random information is 
lost, is particularly subtle: none of the properties we have discussed above in detail needs to remain true if the level of conditioning changes in this way. This message could be taken as a disappointment to a mathematician-certainly, a number of natural conjectures regarding the preservation of a class property turn out to be false. On the other hand, the fact that so much depends on the conditioning stresses the importance of choosing the filtration explicitly. This point is well illustrated by the above Theorem.

Finally we remark about a parallel between our conclusions and the well known "Simpson's paradox" in statistics (see, e.g., Cohen [6]). Stated in simple demographic terms, the crude death rate in country A may be less than that in country B, although every age-specific death rate in country A exceeds the corresponding rate in country $\mathrm{B}$. Here, too, the question is about the distribution of an unknown "underlying" random variable.

\section{References}

[1] ARJAS, E.: A stochastic process approach to multivariate reliability systems: notions based on conditional stochastic order. - Math. Oper. Res. 6, 1981, 263-276.

[2] ArJas, E., and I. Norros: Life lengths and association: a dynamic approach. - Math. Oper. Res. 9, 1984, 151-158.

[3] ArJas, E., and I. Norros: Change of life distribution via a hazard transformation: an inequality with application to minimal repair. - To appear in Math. Oper. Res., 1989.

[4] Barlow, R. E., and F. Proschan: Statistical theory of reliability and life testing. Holt, Rinehart and Winston, New York, 1975.

[5] BrÉmaud, P.: Point processes and queues: martingale dynamics. - Springer Verlag, New York, 1981.

[6] Cohen, J. L.: An uncertainty principle in demography and the unisex issue. - The American Statistician 40, 1986, 32-39.

[7] Norros, I.: Systems weakened by failures. - Stoch. Proc. Appl. 20, 1985, 181-196.

[8] Norros, I.: A compensator representation of multivariate life length distributions, with applications. - Scand. J. Statist. 13, 1986, 99-112.

[9] Norros, I.: The "minimal repair", elimination and externalization of a totally inaccessible stopping time. - Reports of Dept. of Math., University of Helsinki, 1987.

University of Oulu

Department of Applied Mathematics and Statistics

Linnanmaa

SF-90570 Oulu

Finland
University of Helsinki

Department of Mathematics

Hallituskatu 15

SF-00100 Helsinki

Finland 